\title{
TEXTURAL FRACTOGRAPHY
}

\author{
HYNEK LAUSCHMANN, ONDŘEJ RÁČEK, MiCHAL TŮmA AND IVAN NEDBAL \\ Czech Technical University, Faculty of Nuclear Sciences \& Physical Engineering, Dept. of Materials \\ Trojanova 13, 12000 Praha 2, Czech Republic \\ e-mail: lausch@kmat.fjfi.cvut.cz \\ (Accepted May 17, 2002)
}

\begin{abstract}
The reconstitution of the history of a fatigue process is based on the knowledge of any correspondences between the morphology of the crack surface and the velocity of the crack growth (crack growth rate CGR). The textural fractography is oriented to mezoscopic SEM magnifications (30 to 500×). Images contain complicated textures without distinct borders. The aim is to find any characteristics of this texture, which correlate with CGR. Pre-processing of images is necessary to obtain a homogeneous texture. Three methods of textural analysis have been developed and realized as computational programs: the method based on the spectral structure of the image, the method based on a Gibbs random field (GRF) model, and the method based on the idealization of light objects into a fibre process. In order to extract and analyze the fibre process, special methods - tracing fibres and a database-oriented analysis of a fibre process - have been developed.
\end{abstract}

Keywords: database, fatigue, fibre process, Fourier transform, fractography, Gibbs random field, regression, texture.

\section{INTRODUCTION}

The main task of quantitative microfractography of fatigue failures is the reconstitution of the history of a fatigue crack growth process. Specimens of the material are loaded in the laboratory under service conditions and the crack growth process is recorded. Fracture surfaces are documen-ted by SEM and images are studied to relate some information present in the morphology of the crack surface to the macroscopic crack velocity (crack growth rate CGR). So a basis is obtained on which an unknown CGR can be estimated from the fracture surfaces of real parts. Finally, the crack growth process is reconstituted using integration of CGR along the crack growth direction.

The traditional method is based on striations, fine equidistant grooves in the fracture surface (Nedbal et al., 1989; 1997a,b; Kunz et al., 1993). They belong to fractographic features - strictly defined measurable objects in the morphology of the fracture surface. The method cannot be used when striations are not visible, typically due to corrosion (Siegl and Matocha, 1997; Nedbal et al., 2000).

As an alternative, a textural method has been developed in our department since about 1990. Structures in images of fracture surfaces are studied as image textures. The texture is a random structure of similar elements with some kind of ordering. The main problem in fractography consists in a continuous brightness scale and an absence of distinct borders of textural elements.

For the application of the textural method, the mezoscopic dimensional area with SEM magnifications between macro- and microfractography (about 30 to $500 \times)$ is especially suitable. These magnifications were not used very much in the past for the absence of measurable objects in images. The magnification to be used is limited by several conditions related to individual images, to the whole set of images and to image discretization. Images must be pre-processed to obtain a homogeneous (transition invariant) texture, which is convenient for analysis.

Within the textural method, fractographic information is extracted in the form of integral parameters of the whole image. Two general approaches to the analysis have been studied:

1. Estimation of statistical or model parameters directly from gray-scale images without analysis of textural elements. Two methods will be shown based on spectral characteristics (Fourier transformation) and gray-level coincidences (Gibbs random field model). 
2. Extraction of textural elements followed by the application of binary random field models. A method aimed at fibre similar objects will be described.

\section{EXPERIMENT}

Methods developed will be shown in an application to fatigue fracture surfaces of four laboratory specimens (C16 to C19) of stainless steel AISI 304L used in nuclear power plants (Nedbal et al., 2000). CT (compact tension) specimens (Fig.1) were loaded by a constant triangular cycle with $F_{\min }=1450 \mathrm{~N}, F_{\max }=4850 \mathrm{~N}$, frequency $f=1 \mathrm{~Hz}$ in water at $20^{\circ} \mathrm{C}$.

Fatigue crack surfaces were documented using SEM with magnification $200 \times$. The sequence of the images was located in the middle of the crack surface (Fig. 1) and the images were distanced by $0.4 \mathrm{~mm}$. The direction of the crack growth in the images is bottom-up. The real area of one image is about 0.6 $\times 0.45 \mathrm{~mm}$. A digital representation in $1200 \times 1600$ pixels and 256 brightness values was used. The total number of images was 165 . An example of a typical texture is shown in Fig. 2.

From frequently repeated records of the crack length the estimates of CGR were computed. The dependence of the CGR [expressed in $\mu \mathrm{m}$ per loading cycle] on crack length [expressed in $\mathrm{mm}$ ] was estimated and every image was assessed a value of the CGR pertinent to its middle.

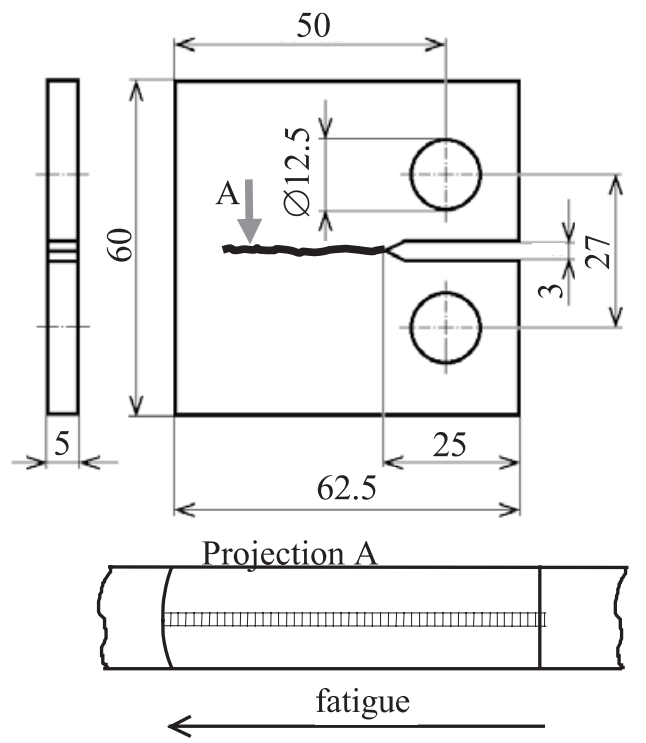

Fig. 1. Specimen for fatigue tests and image location in fatigue crack surface.

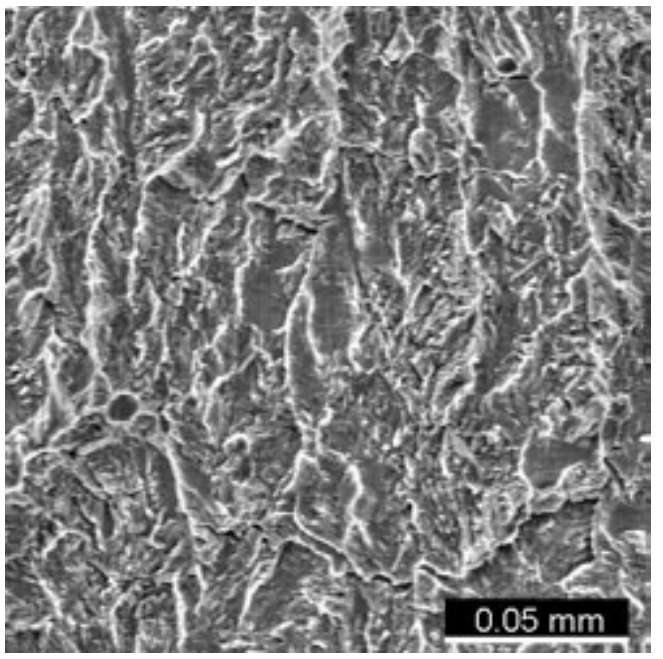

Fig. 2. An example of a typical texture in an ima ge of crack surface (detail $500 \times 500$ pixels).

\section{GENERAL COMMENTS}

SETTING SEM MAGNIFICATION. An appropriate magnification must be optimized with respect to several requirements:

Within one image:

- the number of textural elements is representative enough to characterize the texture objectively,

- the texture is approximately homogeneous (the change with increasing CGR is negligible),

- the change of CGR within one image is negligible (it can be characterized by a constant).

Within the set of images:

- the general character of all textures is the same (the same type of analysis can be applied),

- some feature of the texture is significantly dependent on CGR.

\section{Within the given image discretization:}

- textural elements supposed to be the source of information are well represented.

In practice, these requirements are counteracting and a compromise must be sought.

PRE-PROCESSING OF IMAGES is necessary in order to remove significant fluctuations of mean brightness and contrast. These fluctuations are caused by morphological aspects (height and slope of crack surface) with characteristic dimensions corresponding to the image size or larger, and cannot be left to influence the results. A suitable method normalization (Lauschmann, 2000) - was derived by 
generalization from one-dimensional stochastic processes. Brightness is transformed by a moving algorithm to mean value 128 and standard deviation 50 (for an 8-bit range). In contrast to the generally used equalization (Parker, 1996), the shape of textural elements is conserved. The size of the mask is a very important parameter fundamentally affecting the results. A too small mask destroys textural elements to smaller ones, while a too large mask does not acquire the desired effect.

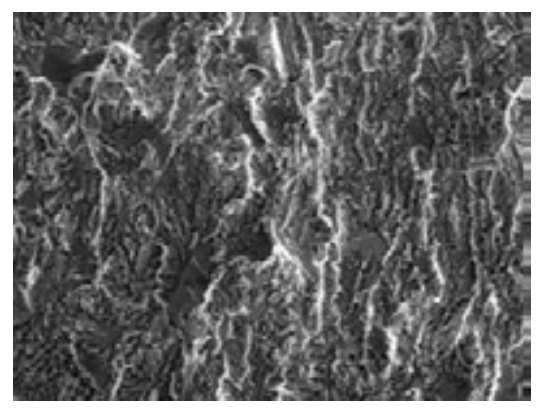

a)

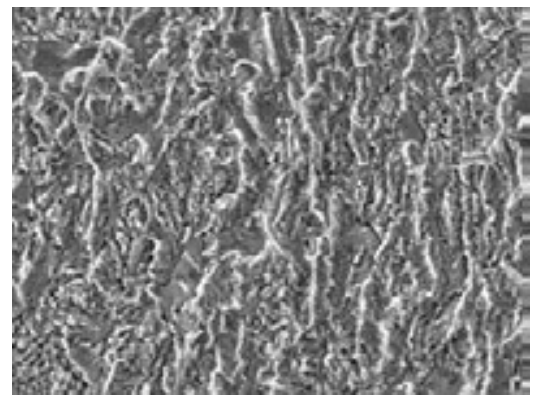

b)

$0.1 \mathrm{~mm}$

Fig. 3. a) original and b) normalized image (section $600 \times 450$ pixels $)$.

MULTILINEAR MODEL. The experience showed that a single image characteristic of any type is not robust enough to characterize the relation between CGR and the crack morphology. Although the dependence can be very close within a set of images from one crack surface, on other crack surfaces (from different testing specimens) the same parameter can depend on CGR in a different way. Therefore, we estimate a set of image characteristics of the given type (for instance spectral) for every image, and this whole set is related to the value of CGR. Due to the range of crack velocity covering several decadic orders, its logarithm $\log _{10} v$ must be considered.

Let us have a set of $q$ images with assessed crack rates $v_{i}, i=1,2, \ldots, q$, and characterized by a set of $k$ textural parameters $f_{u i}, u=1,2, \ldots, k$. The simplest model (Lauschmann et al., 2000) expressing the CGR as a function of a set of image parameters is a multilinear function resulting into a system of regression equations

$$
\overline{\log v_{i}}=\sum_{u=1}^{k} c_{u} f_{u i}+c_{k+1} .
$$

The set of parameters $c_{u}$ can be estimated by the least squares method. The system must be strongly overdetermined - the number of equations (that matches the number of images) must be significantly greater then the number of estimated constants, $\mathrm{q}>>\mathrm{k}+1$.

Not all characteristics $f_{u}$ predicate the CGR. An instrument for testing the significance is the test of the zero value of the estimated coefficients $c_{u}$, $u=1, \ldots, k+1$ (Bakytová et al., 1986). We test the hypothesis $H_{0}: c_{u}=0$ against the alternative $H_{1}$ : $c_{u} \neq 0$. The test criterion is a Student's $t$-distributed statistic

$$
t_{u}=c_{u}\left(\frac{\sum(\log v-\overline{\log v})^{2}}{q-k-2}\left(\left(\boldsymbol{F}^{\prime} \boldsymbol{F}\right)^{-1}\right)_{u, u}\right)^{-1 / 2} .
$$

If the absolute value of $t_{u}$ is lower than the critical value at the selected level of significance $\alpha$ and $q-k-2$ degrees of freedom, $\left|t_{u}\right|<t_{1-\alpha / 2}(q-k-2)$, hypothesis $H_{0}$ cannot be rejected and parameter $f_{u}$ should be excluded.

A handicap originates from the multiparametric character of the method. The model counterbalances many increments from different sources of information. Therefore, a fractographic interpretation of the variety of textural elements is hardly possible.

\section{METHODS}

\section{SPECTRAL ANALYSIS}

The 2D Fourier transformation (Pratt, 1978) is a decomposition of the image matrix $\boldsymbol{X}$ of the size $m \times n$ into a linear combination of basic vectors $\boldsymbol{B}^{r, s}$

$$
\boldsymbol{X}=\sum_{r=0}^{m-1} \sum_{s=0}^{n-1} y_{r, s} \boldsymbol{B}^{r, s},
$$

where

$$
y_{r, s}=\boldsymbol{X}^{*} \boldsymbol{B}^{r, s}=\sum_{k=1}^{m} \sum_{l=1}^{n} x_{k, l} b_{k, l}^{r, s}
$$

A basic vector is a matrix of the same dimensions $m \times n$ which represents a harmonic wave (Fig. 4) with frequency $r$ in the column direction and $s$ in the row 
direction, expressed by the number of periods within the image dimensions. The set of coefficients $y_{r, s}$ creates a complex matrix $\boldsymbol{Y}$ of the same size as the image. The presence of single frequencies without respect to wave phases is expressed by the set of amplitudes called spectrum, $\boldsymbol{A}=|\boldsymbol{Y}|$.

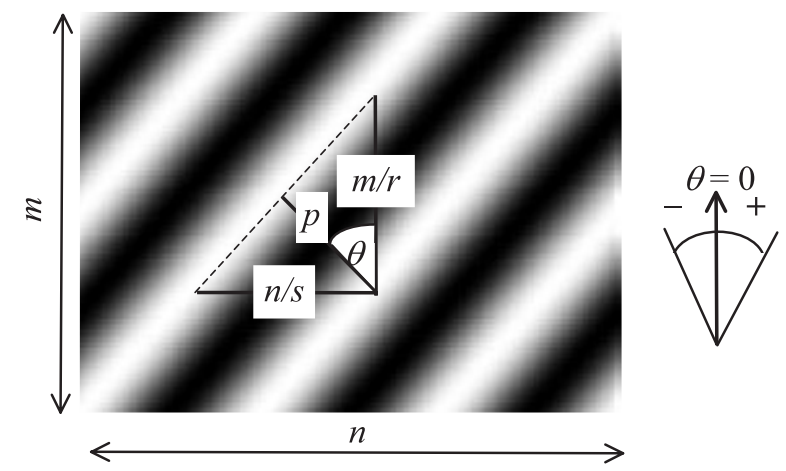

Fig. 4. Example of a planar harmonic wave $-\boldsymbol{B}^{2,3}$ (cosine part, rescaled and shifted).

For the fractographic interpretation, suitable characteristics are not frequencies but distances and directions $(\theta=0$ matches the direction bottom-up in the image). That is why we interpret the spectrum in variables [period, direction] $=[p, \theta]$ (Lauschmann et $a l ., 2000)$. Basic vector $\boldsymbol{B}^{r, s}$ represents a harmonic wave with period (wave length) $p_{r, s}$ and normal direction (direction of the wave vector) $\theta_{r, s}$ (see Fig. 4)

$$
\begin{gathered}
p_{r, s}=\left(\frac{r^{2}}{m^{2}}+\frac{s^{2}}{n^{2}}\right)^{-1 / 2}, \\
\theta_{r, s}=-\operatorname{arctg}\left(\frac{m s}{n r}\right) .
\end{gathered}
$$

In order to reduce the number of output spectral characteristics, a reasonable sorting of both parameters can be introduced. Single segments of the spectrum defined by the Cartesian product of period and direction intervals, $[p, \theta] \in\left(p_{i}, p_{i+1}\right) \times\left(\theta_{j}, \theta_{j+1}\right)$, can be characterized by the mean spectrum

$$
f_{i, j}=\sum_{r, s} a_{r, s} \delta_{r, s} / \sum_{r, s} \delta_{r, s},
$$

where

$$
\delta_{r, s}=\left\langle\begin{array}{l}
\text { lif } p_{r, s} \in\left\langle p_{i}, p_{i+1}\right), \theta_{r, s} \in\left\langle\theta_{j}, \theta_{j+1}\right), \\
\text { 0otherwise. }
\end{array}\right.
$$

APPLICATION [8]. In the case mentioned above, sorting of periods was defined in real distances by interval borders $p=\{1,2,3,4,5,6,8,10,12,14,16$,
$20,24,30\} \mu \mathrm{m}$. Sorting of directions was limited to 3 classes: directions close to the direction of crack growth $\theta \in(-15,15)^{\circ}$, directions close to crack front $\theta$ $\in(-90,-75)^{\circ} \cup(75,90)^{\circ}$ and all other directions. All combinations created 45 segments. Within the application of multilinear regression (1), 19 segments of spectrum have been found to be significant at the level of significance $\alpha=0.05$.

A comparison of experimental CGR taken for the input and CGR values returned from the spectral image characteristics is shown in Fig. 5. The relations of crack length vs. the number of loading cycles are illustrated in Fig. 6. One point represents one image.

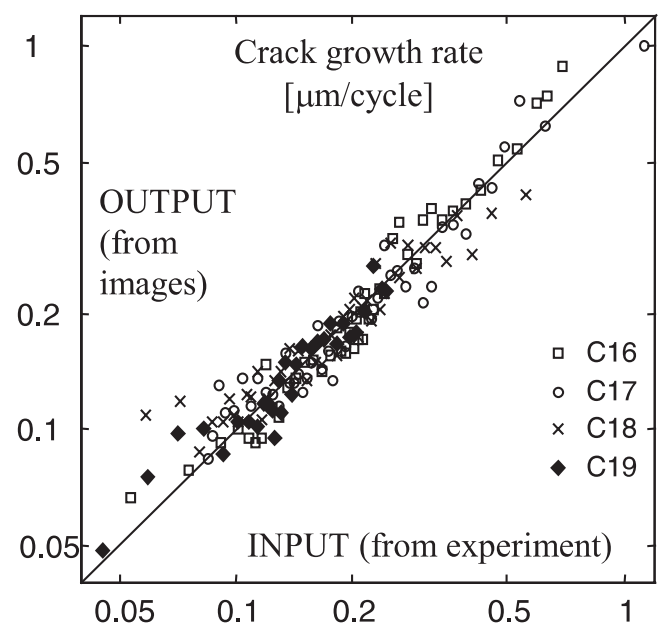

Fig. 5. Comparison of crack rates taken for input and returned from spectrum of images of crack surfaces. Ideal agreement: $y=x$.

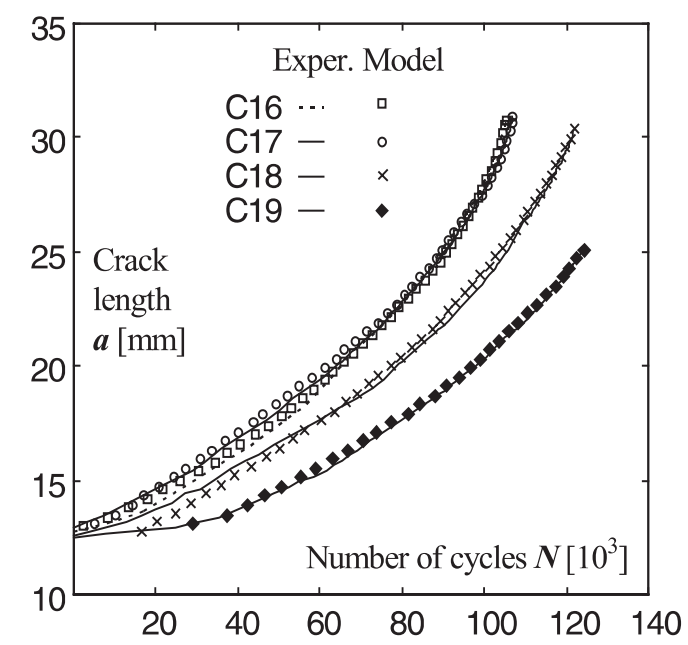

Fig. 6. Crack growth in single specimens and reversal reconstitution (from the end to the origin) using crack rates estimated from images. 


\section{GIBBS RANDOM FIELDS}

A simple GRF model (Gimel'farb, 1999; Lauschmann and Ráček, 2001) of a texture reflects gray levels $x(r, c)$ in single pixels, and interactions differences $d=x_{1}-x_{2}$ between gray levels of two pixels. All pixel pairs with the same distance vector $[i, j]=\left[r_{1}-r_{2}, c_{1}-c_{2}\right]$ create a clique. A list of cliques taken into account is called search window $\boldsymbol{W}$. The distance vector $[0,0]$ is associated with gray levels in single pixels. At random textures, the significance of interactions decreases with the increasing distance between the pixels creating the pair.

The main sample characteristic of an image $\boldsymbol{X}$ is the gray level co-occurrence histogram $\boldsymbol{h}(\boldsymbol{X}) . h_{i, j, d}$ is the number of interactions $d$ in the clique $[i, j]$. Probability of interactions is $p_{i, j, d}=h_{i, j, d} /[(m-|\mathrm{i}|)(n-$ jj)], where $m, n$ are image dimensions.

The main GRF characteristic is potential $V$, a three-dimensional structure $\left\{V_{i, j, d}\right\}$ of the same size as $\boldsymbol{h}$. It compresses the information on gray level cooccurrences in the way similar to the energy of interactions of particles in statistical physics. Within a random field (space of images) defined by potential $\boldsymbol{V}$, two probability distributions are especially important:

Gibbs probability distribution of image $\boldsymbol{X}$ equals (Gimelfarb, 1999)

$$
\operatorname{Pr}(\boldsymbol{X})=\frac{\exp \left(\boldsymbol{V}^{*} \boldsymbol{h}(\boldsymbol{X})\right)}{\sum_{\xi \in \Xi} \exp \left(\boldsymbol{V}^{*} \boldsymbol{h}(\xi)\right)},
$$

where * denotes a scalar product of arrays $\boldsymbol{V}$ and $\boldsymbol{h}(\boldsymbol{X})$ reshaped into vectors. The denominator is a normalization constant with $\Xi$ denoting the set of all possible images.

The distribution of the probability of brightness in a pixel $[r, c]$, given brightness values in all interacting pixels, is expressed by (Gimelfarb, 1999)

$$
\begin{gathered}
\operatorname{Pr}(x(r, c) \mid x(r+i, c+j),[i, j] \in \mathbf{W} \backslash[0,0])= \\
\exp \sum_{[i, j] \in \mathbf{W} \backslash[0,0]} \mathbf{V}_{i, j, x(r, c)-x(r+i, c+j)} \\
\sum_{u \in U} \exp \sum_{[i, j] \in \mathbf{W} \backslash[0,0]} \mathbf{V}_{i, j, u-x(r+i, c+j)}
\end{gathered}
$$

where $U$ denotes the set of all possible values of brightness.

For a given image $\boldsymbol{X}_{\boldsymbol{o}}$, potential $\boldsymbol{V}$ can be estimated in two steps: The first approximation based on Taylor expansion of the likelihood function based on (Eq. 6) is refined by means of stochastic relaxation using (Eq. 7). Within it, the difference between the training sample $\boldsymbol{X}_{\boldsymbol{o}}$ and current sample $\boldsymbol{X}^{(t)}$ is measured by chi-square distance

$$
\Delta\left(\mathbf{X}_{\mathbf{0}}, \mathbf{X}^{(t)}\right)=\sum_{[i, j] \in \mathbf{W}} \sum_{d \in \mathbf{D}}\left(p_{i, j, d}\left(\mathbf{X}_{\mathbf{o}}\right)-p_{i, j, d}\left(\mathbf{X}^{(t)}\right)\right)^{2}
$$

In the course of relaxation, the difference $\Delta$ decreases to a constant and its fluctuations to zero. In this way we obtain an estimate of the potential together with a model texture $\boldsymbol{X}^{(t)}$ which was optimized to be close to the image. In Fig. 7, original and model textures are compared. Although visually the agreement is not very satisfactory, the results obtained are excellent.

A suitable measure of the significance of cliques $[i, j]$ is their relative energies (Fig. 8)

$$
e_{i, j}=\sum_{d \in D} V_{i, j, d} p_{i, j, d}\left(X_{0}\right),[i, j] \in \boldsymbol{W},
$$

from which only those for small distance components $i, j$ are significant. A set of significant relative energies reshaped into vector $\boldsymbol{f}$ can be used for image characteristics to be applied in the multilinear re ${ }^{\text {ression }}$ (1).

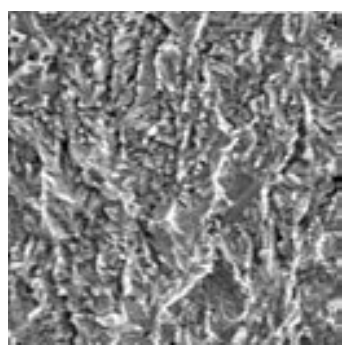

1a

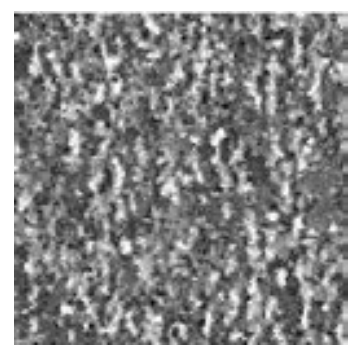

1b

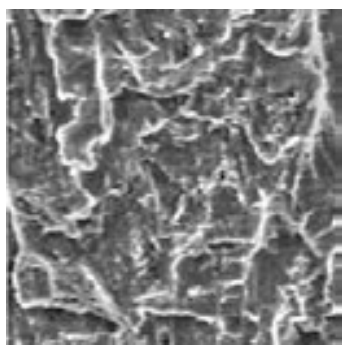

2a

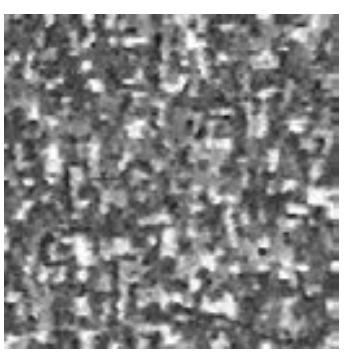

2b

Fig. 7. Comparison of original (a) and model (b) textures. Small (1) and high (2) CGR. Section $256 \times 256$ pixels. 


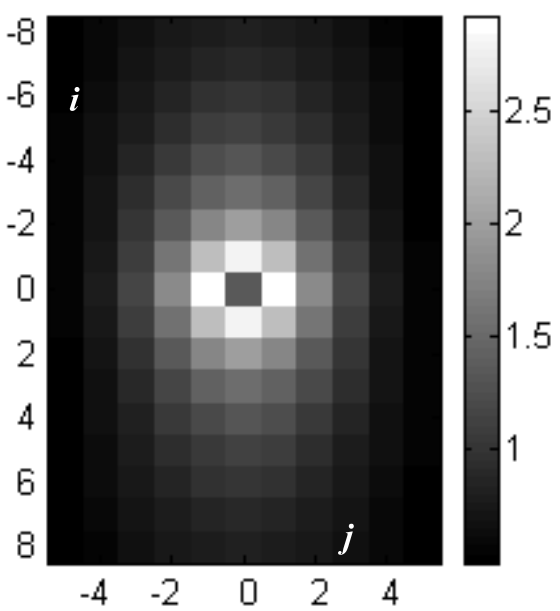

Fig. 8. An example of relative energies $e_{i, j}$ - region of significant values.

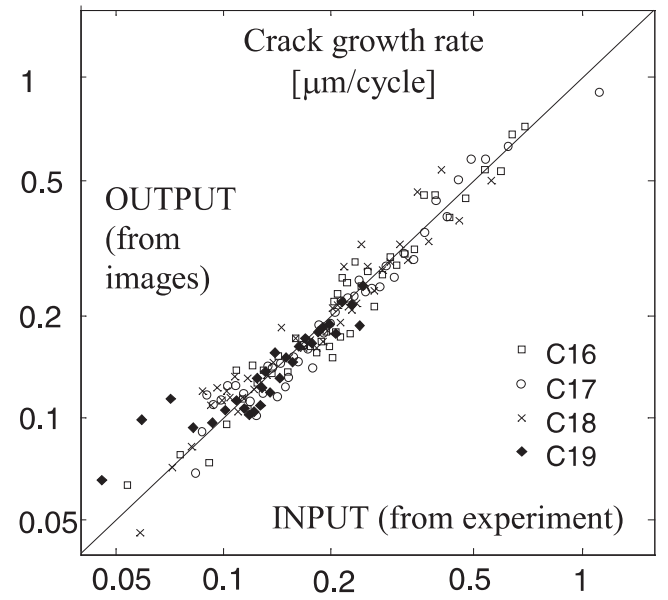

Fig. 9. Comparison of crack rates taken for the input and returned from the GRF model of image texture.

APPLICATION. 31 energies $e_{i, j}$ were chosen that were significant in all images. 25 of them were found to be significant in the multilinear regression at the level of significance $\alpha=0.05$. The result is plotted in Fig. 9. One point represents one image. The relation of the crack length vs. the number of cycles is not visibly different from that in Fig. 6.

\section{FIBRE PROCESS}

In many cases, the most remarkable elements of textures are light fibres with a different thickness and shape. They reflect sharp ridges and edges in the fracture surface. This structure can be abstracted into a fibre process (Čejka and Beneš, 1999; Lauschmann, 2000), the properties of which are studied to find any sensitivity to the crack growth velocity. The length of continunus fihres is an imnortant nronerty The requirement to analyze the continuity of fibres in points of crossing or branching ("knots") made it necessary to create a database of the fibre process. Consequently, it was found that such a database could be used to estimate many important characteristics of the process.

First we will show how fibre processes may be extracted from fractographic images, and then the creation and exploitation of the database will be described.

EXTRACTION OF FIBRES. Images were normalized to receive a homogeneous texture (Fig. 11a). The binarization by threshold met troubles because received objects did not correspond with qualities of fibres at any value of the threshold. A procedure, deliberately aimed at light fibre objects, was proposed in following steps (Lauschmann et al., 2001a; Lauschmann, 2001):

1. Marr \& Hildreth edge detection (Parker, 1996) modified on fibres. The basis is a convolution mask

$$
\boldsymbol{U}_{2,5}=\left[\begin{array}{lllll}
-1 & -3 & 9 & -3 & -1 \\
-1 & -3 & 9 & -3 & -1
\end{array}\right]
$$

which enhances a vertical light fibre of 1 or 2 pixels in width. An example of its effect is shown in Fig. 10.

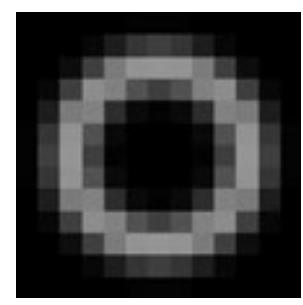

a

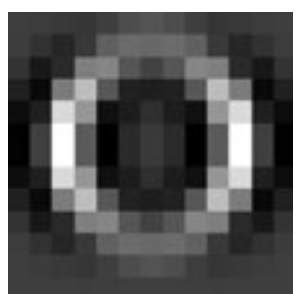

b
Fig. 10. Illustration of elementary fibre detection. a) a fibre without distinct borders in gray-scale image. b) result of filtering by $\boldsymbol{U}_{2,5}$ (rescaled).

By rescaling the mask into $\boldsymbol{U}_{r, s}$ the effect can be optimized to a fibre width of about $s / 3$ and a direct element length of $r$ pixels. In our case,

$$
\begin{aligned}
& \boldsymbol{U}_{5,12}= \\
& =\left[\begin{array}{cccccccc}
-1 & -2.17 & -3.41 & -2.34 & 2.86 & 8.09 & \\
\vdots & \vdots & \vdots & \vdots & \vdots & \vdots & \\
& 8.09 & 2.86 & -2.34 & -3.41 & -2.17 & -1 \\
& \vdots & \vdots & \vdots & \vdots & \vdots & \vdots
\end{array}\right]
\end{aligned}
$$

was rotated by $0, \pi / 8, \ldots, 7 \pi / 8$ and the maximum of all the annlications was taken for the result (Fig. 11h). 


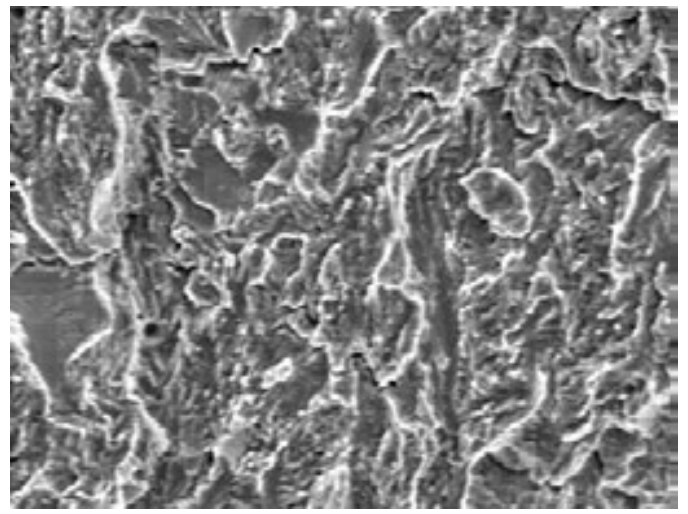

a)

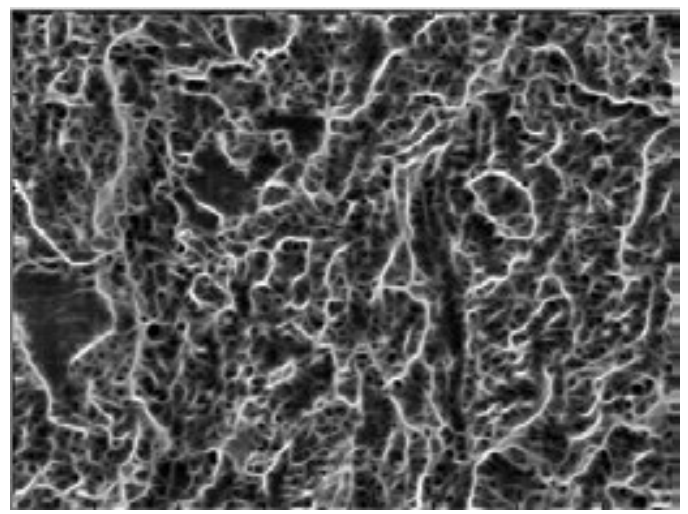

b)

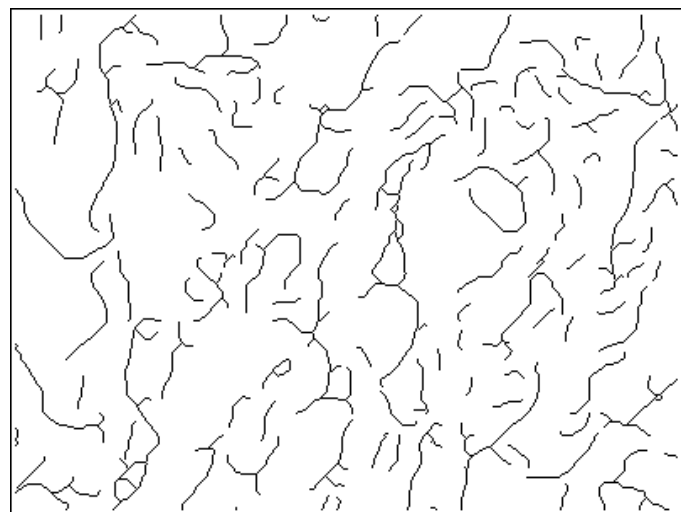

c)

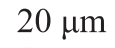

Fig. 11. Extraction of light fibres from an image of the fatigue crack surface. a) normalized image (detail $400 \times 300$ pixels), b) fibre detection by $\boldsymbol{U}_{5,12}$ (rescaled into 8 bit range), c) significant fibres traced.

2. From the starting points - the lightest pixels in the image - fibres are traced towards both sides using a set of directional modifications of the mask

$$
V_{0}=\left[\begin{array}{ccccccc}
0 & 0 & 0.5 & 0.5 & 1 & 1 & 1 \\
\{1\} & 1 & 1 & 1 & 1 & 1 & 1 \\
0 & 0 & 0.5 & 0.5 & 1 & 1 & 1
\end{array}\right],
$$

where $\{1\}$ denotes the position of the last recognized crack pixel. The direction related to the maximal result of convolutions is accepted for the next step of tracing the fibre.

To avoid double tracing or reversal progress, recognized fibres are prohibited for further processing in the width of 7 pixels. Tracing finishes when brightness in the last pixel found does not reach a selected limit. Short parts of fibres close to knots are supplemented by a special algorithm (Lauschmann et al., 2001a). Very small objects of the skeleton are excluded, as they represent small nonfibrous patches (Fig. 11c).

FIBRE PROCESS ANALYSIS AND CREATION OF DATABASE (Lauschmann, 2001). For any description of a fibre process, the binarized image must be analyzed into elements. Three types of them are recognized: individual fibres, vertices and objects. Vertices are endpoints of fibres. Two types of vertices can be distinguished: isolated fibre endpoints and knots - common endpoints of several fibres. An object is a continuous set of fibres.

Recognition of objects is a separate problem, which can be solved by using generally recommended algorithms (e.g, the procedure bwlabel in Matlab's Image Processing Toolbox).

In order to classify pixels of the process as internal points, knots or endpoints, the number of changes background $\rightarrow$ fibre along a curve surrounding every pixel in a given distance can be used, as it is shown in Fig. 12. Simultaneously, the number of fibres leaving every vertex is obtained. The set of vertices is to be renumbered by their pertinence to objects. The following step of the analysis consists in recording fibres from the starting to the finishing vertex. The simplest way is to check the neighborhood of the size $3 \times 3$ of the last recognized pixel, while all pixels of the process recognized up to now are prohibited to avoid reversal steps. At first, row and column directions must be checked, and if no pixel has been found, also diagonal directions should be tested (Fig. 13) 


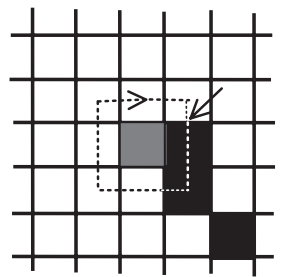

a

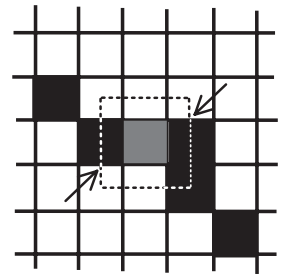

b

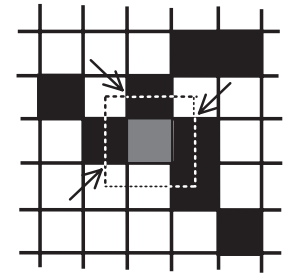

c

Fig. 12. Testing the type of pixels of the process by the number of changes background $\rightarrow$ fibre along a curve surrounding the pixel. a) $n=1$, endpoint, b) $n=2$, internal fibre point, c) $n$ $>2$, knot.

During the analysis, the database components are gradually filled in. The database structure was proposed so that it offers extracting information on objects and long fibres without browsing. The database can be implemented in ordinary programming languages. For computing characteristics of a higher order (e.g. mutual relations of fibres), the database should be exported to a special database platform, where elements of a given set of properties can be quickly selected. Although the database contains a complete description of the process and its structure, the compression is $80 \%$ and higher in comparison to a bitmap.

Within every fibre, starting and finishing vertices are chosen and distinguished. Vertices are organized by objects and fibres by their starting vertices. Therefore also the sequence of fibres is organized by objects.

Let $n_{o}, n_{v}, n_{f}$ and $n_{p}$ be numbers of objects, vertices, fibres and all pixels of the process, respectively. The database consists of four matrices: the list of objects $\boldsymbol{O}$, size $\left[n_{o}, 1\right]$; the list of vertices $\boldsymbol{V}$, size $\left[n_{v}, 5\right]$; the list of fibres $\boldsymbol{F}$, size $\left[n_{f}, 4\right]$; and the list of internal pixels of fibres, size $\left[n_{p}-n_{v}, 2\right]$. An example of the database is shown in Fig. 14.

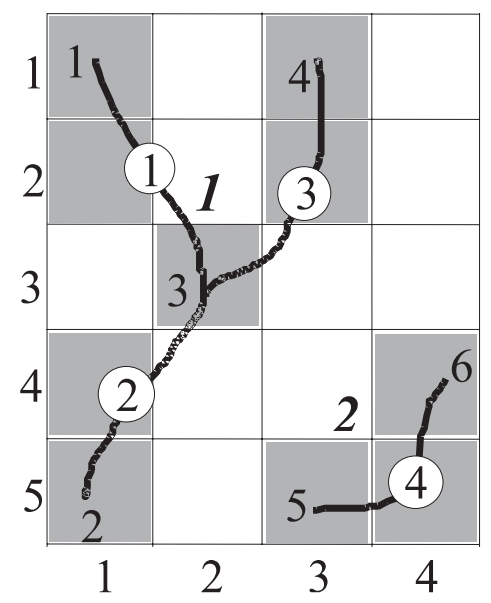

Objects:

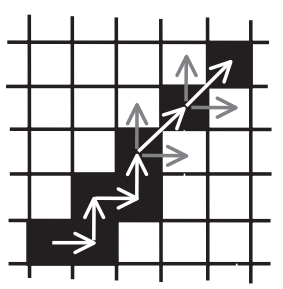

Fig. 13. Tracing a fibre with preference of row and column direction.

\section{1,2}

$$
n_{o}=2
$$

Fibres: (1), 2), 3, (4) $n_{f}=4$

Vertices: $1,2,3,4,5,6 \quad n_{v}=6$

Pixels:

$$
n_{p}=10
$$

$\boldsymbol{O}$ - list of objects

$$
\begin{aligned}
& \text { the first vertex of the given object } \\
& \hline O(1)=1 \\
& O(2)=5
\end{aligned}
$$

\begin{tabular}{|c|c|c|c|}
\hline object & row & column & 1 \\
\hline$V(1,:)=[1$ & 1 & 1 & 1 \\
\hline$V(2,:)=[1$ & 5 & 1 & 2 \\
\hline$V(3,:)=[1$ & 3 & 2 & 3 \\
\hline$V(4,:)=[1$ & 1 & 3 & 0 \\
\hline$V(5,:)=[2$ & 5 & 3 & 4 \\
\hline$V(6,:)=[2$ & 4 & 4 & 0 \\
\hline
\end{tabular}

$V$ - list of vertices

$\boldsymbol{F}$ - list of fibres

$\left.\begin{array}{cccc}\text { vertex start } & \text { end } & 3 / & 4 / \\ \hline F(1,:)=\left[\begin{array}{llll}1 & 3 & 1 & 1\end{array}\right] \\ F(2,:)=[2 & 3 & 2 & 2] \\ F(3,:)=[3 & 4 & 3 & 3] \\ F(4,:)=[5 & 6 & 4 & 4\end{array}\right]$

$\boldsymbol{P}$ - list of internal pixels of fibres

\begin{tabular}{ll} 
row & column \\
\hline$P(1,:)=\left[\begin{array}{ll}2 & 1\end{array}\right]$ \\
$P(2,:)=\left[\begin{array}{ll}4 & 1\end{array}\right]$ \\
$P(3,:)=\left[\begin{array}{ll}2 & 3\end{array}\right]$ \\
$P(4,:)=\left[\begin{array}{ll}5 & 4\end{array}\right]$
\end{tabular}

$1 /$ The first fibre starting in the given vertex.

2/ The first fibre on list $F(:, 4)$, finishing in the given vertex.

$3 /$ The first internal pixel of the given fibre on list $\boldsymbol{P}$.

4/ The list of fibres ordered by finishing vertex.

Fig. 14. An example of a fibre process database 
PARAMETRIC MODEL AND JOINING LONG FIBRES IN KNOTS (Lauschmann et al., 2001a). Let $\left[x_{t}, y_{t}\right], t=1,2, \ldots, n$ be the coordinates of fibre pixels in the sequence from the starting to the finishing vertex. A moving parametric regression can be used with the length of a single regression $T$ and the shift of moving about $T / 3$ (only the central third of the regression is accepted). For the regression function, we used a combination of the polynomial and polyharmonic models (corresponding to Taylor and Fourier decomposition) in the elementary form

$$
\begin{aligned}
& \bar{x}(t)=a_{0 j}+a_{1 j} \tau+a_{2 j} \sin (\varphi)+a_{3 j} \sin (2 \varphi), \\
& \bar{y}(t)=b_{0 j}+b_{1 j} \tau+b_{2 j} \sin (\varphi)+b_{3 j} \sin (2 \varphi), \\
& \text { where } \tau=t-t_{0 j}+1, \varphi=\pi \tau / T
\end{aligned}
$$

Here $j$ denotes the present recurrence of the regression and $t_{0 j}$ is its starting index. The linear component of Eq. 13 expresses the position and main course of the $j$-th segment, while the trigonometric components express its "waving". A single regression does not have more than one inflection. In consequence, the maximum density of regression inflections is one for $T / 3$ pixels of the fibre. This ratio defines the smoothing of details, which can be selected by setting $T$ with respect to the aims of the analysis.

In every knot, all possible pairs of fibres are checked whether they create a longer fibre passing the knot. A common regression of merged fibres is estimated and its curvature in the knot serves for the decision. If it is smaller than a selected threshold, both fibres are judged as creating a passing one. This information is implemented in the database as 2 columns added to matrix $\boldsymbol{F}$, where, for fibre $k$,

$\boldsymbol{F}(k, 5)$ is the number of the fibre connected in the starting vertex,

$\boldsymbol{F}(k, 6)$ is the number of the fibre connected in the finishing vertex.

The absence of any connection is expressed by zero.

Final regressions are computed in the same manner as described above, for joined fibres. Computing is so quick that it is not efficient to store regressions in database structures.

\section{ESTIMATION OF FIBRE PROCESS} CHARACTERISTICS (Lauschmann et al., 2001a). The parametric model of a fibre process makes possible the estimation of a wide class of properties. Characteristics of length. direction. nosition and without any problems with the discrete image representation. In many cases, a sufficient source for estimation characteristics are points of regressions $[\bar{x}, \bar{y}](13)$, that are pertinent to single pixels of binary skeletons of fibres. Increments between them can be used to estimate distributions of lengths and orientations:

$$
\begin{gathered}
\Delta l_{t \rightarrow t+1}=\sqrt{(\bar{x}(t+1)-\bar{x}(t))^{2}+(\bar{y}(t+1)-\bar{y}(t))^{2}}, \\
\phi_{t \rightarrow t+1}=\operatorname{arctg}\left(\frac{\bar{y}(t+1)-\bar{y}(t)}{\bar{x}(t+1)-\bar{x}(t)}\right) .
\end{gathered}
$$

Also more complicated qualities can be studied for example the joint distribution of lengths and orientations (Fig. 15), the random process of the fibre orientation along its length, etc. Many of these characteristics would not be available by traditional methods.

APPLICATION (Lauschmann et al., 2001b). For texture characteristics, the joint distribution of fibre length and orientation (Fig.15) was used. The sorting was roughened into four classes for the direction $\left(\theta \in(-10,10)^{\circ},(-40,-10\rangle^{\circ} \cup\langle 10,40)^{\circ},(-70,-40\rangle^{\circ} \cup\right.$ $\left.\langle 40,70)^{\circ},(-90,-70\rangle^{\circ} \cup\langle 70,90)^{\circ}\right)$ and six classes for the length (defined by class borders 10-30-50-70100-140-500 pixels). Of resulting 24 image parameters, 18 have been found to be significant in the multilinear regression at the level of significance $\alpha=0.05$. Results are documented in Fig. 16. One point represents one image. The relation of the crack length vs. the number of cycles is similar to that in Fig. 4.

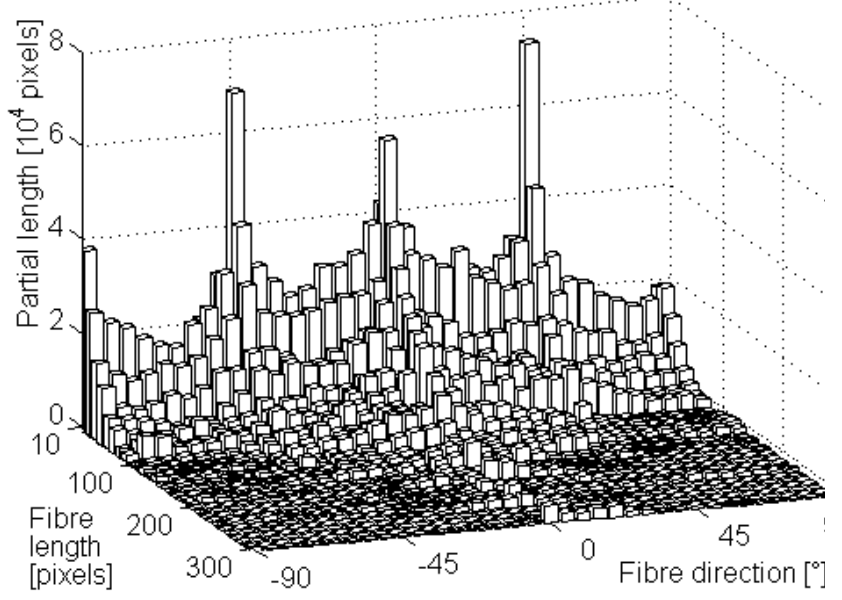

Fig. 15. An example of joint distribution of the lengths and orientations of light fibres from one 


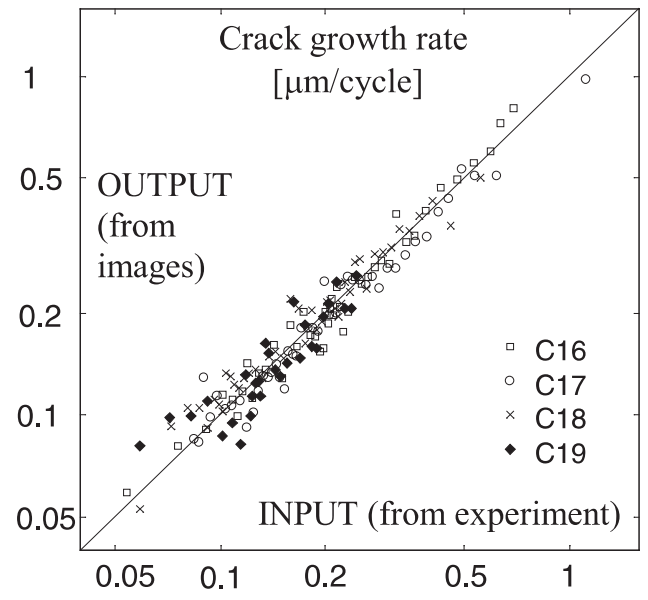

Fig. 16. Comparison of crack rates taken for input and returned from the fibre distribution.

\section{CONCLUSIONS}

SEM images of fatigue crack surfaces under magnifications between macro- and microfractography (about 30 to $500 \times$ ) contain a complex image texture, which is a rich source of information on the crack growth process. Three ways of fractographic textural analysis have been developed and brought to practical applicability, including routine computational programs: spectral analysis, modelling texture as a Gibbs random field, and analysis of light fibres. Within all the methods, the velocity of crack growth can be estimated as a multilinear function of a set of numerical characteristics of textures in images of fracture surfaces.

Textural fractography can complement or substitute traditional methods. It opens possibilities to obtain new information from fracture surfaces, which has been concealed up to now. Simultaneously, textural method transfers the main work from the operator to the computer.

Two new methods within the general frame of image analysis have been developed:

- The method of tracing fibres is a practical alternative to creating a skeleton for fibre structures without distinct borders in gray scale images.

- The method of composing a database and a parametric model of a fibre process was proposed to meet special requirements in fractography. However, no special conditions were supposed, so that the method is generally applicable. It has been fully automated and we hope that it could find a wide use in analyses of planar fibre processes, tessellations, etc.

The traditional stereological approach to acquisition of data on a fibre structure by means of testing lines was drawn up to a high theoretical level (e.g., Kärkkäinen and Jensen, 2001). However, the database-oriented method offers some additional possibilities that will be discussed in a separate paper.

\section{ACKNOWLEDGEMENT}

This research has been supported by the Grant Agency of the Czech Republic, project No. 106/00/1715 "Computer aided quantitative fractography of fatigue failures".

\section{REFERENCES}

Bakytová H, Hátle J, Novák I, Urgon M (1986). Statistical induction for economists (in Czech). Prague Bratislava, SNTL - Alfa, 228-38.

Čejka V, Beneš V (1999). Computer Aided Fractography: Methods for evaluation of image anisotropy. In: Int. Conf. on Stereology, Spatial Statistics and Stochastic Geometry. Prague: Union of Czech Mathematicians and Physicists, 89-94.

Gimel'farb GL (1999). Image textures and Gibbs random fields. Dordrecht: Kluwer Academic Publishers, 1250.

Kunz J, Nedbal I, Siegl J, Pártl O (1993). Fractographic Remarks to Fatigue Growth Rate. In: Bailon JP and Dickson IJ, eds. Proc. Fatigue '93 (Montreal). Vol. II,. Cradley Heath: EMAS, 649-54.

Kärkkäinen S, Vedel Jensen EB (2001). Estimation of fibre orientation from digital images. $8^{\text {th }}$ European Congress for Stereology \& Image Analysis, Bordeaux Sept. 3-7, 2001. Image Anal Stereol 20(Suppl 1):420-4.

Lauschmann H, Beneš V (1997). Spatial statistics in material research. In: Kitsos ChP and Edler L, eds. Industrial statistics. Hiedelberg: Physica-Verlag, 285-93.

Lauschmann H (1997). Computer aided fractography. In: Parilák L, ed. Proc. Int. Conf. Fractography '97. Košice: Institute of Materials Research of the Slovak Academy of Sciences, 181-8.

Lauschmann H (1999). Computer aided fractography: The spectral analysis of fatigue crack images. In: Int. Conf. on Stereology, Spatial Statistics and Stoch. Geometry. Prague: Union of Czech Mathematicians and Physicists, 171-6.

Lauschmann H (2000). Textural analysis of fatigue crack surfaces - Image pre-processing. Acta Polytechnica 40:4:123-9.

Lauschmann H, Adámek J, Nedbal I (2000). Textural fractography: Spectral analysis of images of fatigue 
crack surfaces. In: Parilák L, ed. Fractography 2000. Košice: Institute of Materials Research of the Slovak Academy of Sciences, 313-20.

Lauschmann H, Wetzig K, Menzel S, Göbel T (2001a). Fractography of crack networks in thin layers. Materials Characterization 46:105-11.

Lauschmann H, Ráček O (2001). Textural fractography: Application of Gibbs random fields. In: Proc. $3^{\text {rd }}$ Int. Conf. Materials Structure \& Micromechanics of Fracture, Brno June 27-29, 2001. Brno University of Technology,196-205.

Lauschmann H, Tůma M, Ráček O, Nedbal I (2001b). Textural fractography. $8^{\text {th }}$ European Congress for Stereology \& Image Analysis, Bordeaux Sept. 3-7, 2001. Image Anal Stereol 20(Suppl 1):372-8.

Lauschmann H (2001). A database-oriented analysis of a fibre process in fractography. $8^{\text {th }}$ European Congress for Stereology \& Image Analysis, Bordeaux Sept. 3-7, 2001. Image Anal Stereol 20(Suppl. 1):379-85.

Nedbal I, Siegl J, Kunz J (1989). Relation Between Striation Spacing and Fatigue Crack Growth Rate in Al-Alloy Sheets. In: Salama K et al., eds. Advances in Fracture Research (Proc. ICF 7, Houston). Vol. 5. Oxford: Pergamon Press, 3483-91.
Nedbal I et al. (1997a). One lecture on fractography of fatigue failures. École d'Été - Dévelopments Récents en Fatigue des Matériaux et des Structures. SaintPierre d'Oléron, Juin 1997. Châtenay Malabry: École Centrale Paris - ICTM, 1-25.

Nedbal I, Kunz J, Siegl J (1997b). Microfractographic Aspects of Fatigue Crack Growth in 7010 Aluminium Alloy. In: Parilák L, ed. Proc. Int. Conf. Fractography '97. Košice: Institute of Materials Research of the Slovak Academy of Sciences, 264-70.

Nedbal I, Kunz J, Siegl J (2000). Influence of corrosion environment and stress ratio on fatigue crack growth in stainless steel 304 L. In: Parilák L, ed. Fractography 2000. Košice: Institute of Materials Research of the Slovak Academy of Sciences, 293-300.

Parker JR (1996). Algorithms for image processing and computer vision. New York: John Wiley \& Sons, 1417.

Pratt WK (1978). Digital image processing. New York: John Wiley \& Sons, 235-42.

Siegl J, Matocha K (1997). Environmentally Assisted Cracking of 10GNN2MFA Low Alloy Steel. In: Parilák L, ed. Proc. Int. Conf. Fractography '97. Košice: Institute of Materials Research of the Slovak Academy of Sciences, 294-9. 